\title{
ATTRACTIVE DISLOCATION AND PARTICLE INTERACTIONS IN ODS SUPERALLOYS -
}

\author{
AND IMPLICATIONS
}

Alice H. Cooper, Vincent C. Nardone and John K. Tien

Center for Strategic Materials

Henry Krumb School of Mines

Columbia University

New York, New York 10027

$\underline{\text { Summary }}$

For the oxide dispersion strengthened (ODS) alloys, dislocation-particle interaction is generally believed to be repulsive since the elastic constants of the oxide dispersoids are believed to be greater than those of the matrix. High temperature strengthening is thought of in terms of a dislocation climb modified Orowan type bowing mechanism between the particles. Dislocation escape occurs when the stress is sufficient to cause the dislocation to bow to a critical configuration. Contrary to this general belief, through detailed TEM microscopy study of crept and hot tensile tested Inconel MA 754, we have found and will report in this paper that the interaction between dislocation and oxide dispersoid is in fact attractive, with the high temperature properties of ODS alloys stemming from this unexpected attractive interaction. In particular, the characteristic and important threshold behavior of ODS alloys during creep at high temperatures is discussed. It is proposed that the finding of attractive interaction opens new avenues for the alloy design of ODS alloys, including the pinning of dislocations next to the dispersoids by additional clcments and the purposeful introduction of non-adherent interfaces between dispersoids and matrix. In the limit, the high temperature (or low strain rate) threshold stress of, say, Incone1 MA 754 at 1093 deg. C, can be increased by a factor of two from $94 \mathrm{MPa}(14 \mathrm{ksi})$ to $188 \mathrm{MPa}$ (28 ksi). 


\section{Introduction}

Dislocation-particle interactions are of fundamental importance in understanding the deformation behavior of particle strengthened systems. For oxide dispersion strengthened (ODS) mechanically alloyed (MA) alloys, the fine oxide particles are plastically nondeformable. Shearing of the particles by mobile dislocations is then not a viable mode of deformation as is the case with the gamma prime precipitation strengthened superalloys.

Heretofore it is believed that nonshearable particles strengthen through either the classical Orowan mechanism or the Orowan mechanism modified by climb of the dislocations over the obstacles $(1,2)$. Implicit in the Orowan mechanism is that the elastic moduli of the obstacles is higher than that of the metallic matrix, resulting in dislocation and particle repulsion and in the classical Orowan encounter (1) of mobile dislocation bowing between two particles, reaching (in the most simplistic view) the critical configuration of a complete half-loop before becoming unstable, folding back on itself and escaping by leaving an Orowan loop around each particle. Figure 10.1 in reference 3 is a typical TEM representation of the Orowan mechanism caught in actual operation (3) in a system where the elastic moduli of the oxide particle are higher than those of the matrix. At high temperatures, when dislocation climb can occur, it has been shown that now as the mobile dislocation bows, it is also climbing over the particles and by the time it reaches the other side and escapes, the bow will not yet reach the half-loop configuration (2). Since the resisting stress is a direct function of dislocation line tension, which in turn is dependent upon how bowed the mobile dislocation becomes before escape, the strengthening stress in ODS, MA alloys has been taken to be between the Orowan stress and a high temperature (or low strain rate) limiting value of about 0.4 (2) of the appropriate Orowan type stress. Accordingly, traditional thinking would have it that the limiting or threshold strength for ODS-MA alloys at high temperatures would be about 0.4 of the Orowan stress. At first glance this appears to be the case.

A representative example of the high temperature behavior of ODS-MA alloys is given in Fig. 1 for single crystal Ni20Cr $2 \mathrm{ThO}_{2}$ (4) and for high grain aspect ratio Inconel MA $754(5,6)$. Grain boundary effects can be excluded for these systems, so that their high temperature, slow strain rate (i.e. creep) behavior can indeed be attributed to dislocation particle interaction. As shown in Figure 1, ODS alloys possess a characteristic "threshold stress" for dislocation creep, below which no detectable creep occurs. From temperature and modulus compensated yield strength data, Pharr and Nix (7) determined that the modulus compensated Orowan stress for the Lund and Nix system, Fig. 1a, is about 1.7 times greater than the threshold shown in the figure. Similarly, Nardone et al. (6) concluded that the modulus compensated Orowan stress for MA 754, Fig. 1b, is about twice that of the threshold shown in that figure. Petkovic-Luton et al. concluded that for the Inconel MA 956 system that the Orowan stress is also about a factor of two greater than the experimentally determined threshold stress for that ODS-MA alloy (8).

According1y, it does appear that a climb modified Orowan mechanism may determine the limited high temperature creep strength of ODS-MA alloys. In other words, the high temperature threshold strength of an ODS alloy will always be less than about one-half the temperature compensated Orowan stress produced by the interparticle spacings and other geometric features of that alloy. We will show below that this is a false constraint, since the dislocation dispersoid interaction can be an attractive one, and the 
threshold may be increased by a factor of two through appropriate microchemistry and oxide/matrix interface modifications.

\section{Experimenta1}

The alloy chosen for this study was Inconel MA 754 which is essentially a $\mathrm{Ni} 20 \mathrm{Cr}$ solid solution strengthened by $\mathrm{Y}_{2} \mathrm{O}_{3}$ dispersoids; a detailed microstructural characterization has been reported elsewhere by Howson et al. (5). The grain aspect ratio is about 10 . The $\mathrm{Y}_{2} \mathrm{O}_{3}$ fraction is 2.5 percent by volume. The average oxide size is $14 \mathrm{~nm}$, and interparticle spacing was determined to be $110 \mathrm{~nm}$ (5). In order to confirm earlier (5) strain rate versus stress data, and to produce structures for TEM analysis, creep tests were done on a dead-1oad machine and the reported creep rates are \pm 5 percent in accuracy (5). Temperature was maintained to \pm 2 deg. C. In order to obtain higher strain rate data tensile tests were also performed in a computer-controlled MTS machine under constant true strain rate conditions. All mechanical tests were done with specimen axis parallel to the long grain axis to eliminate grain boundary effects during creep.

The representative TFM microstructures investigated were of five specimens tested in the disk (760 deg. C) temperature regime and four specimens tested in the vane or blade (1093 deg. C) temperature regime. About half of the tests were interrupted at the saturation stress (9) under tensile conditions and half in the steady state regime under creep loading conditions. Some specimens were cooled on load by opening the split furnaces. In addition some specimens were crept into steady state, unloaded and allowed to anneal at temperature for one to three hours to determine the lower energy configurations of the dislocations at the particles. TEM foils were prepared parallel to the primary (111) slip plane in most cases. Foils were thinned with a 55\% ethanol, $30 \% \mathrm{n}-$ Butanol and $15 \%$ perchloric acid (70\%) solution and under an applied voltage of $40 \mathrm{~V}$ at -6 deg. C. AII TEM observations were made on a JEOL Model JEM-100CX scanning-transmission electron microscope. Observations of dislocation-particle interaction were usually made over several specimen stage rotations, with tilts as high as 50 degrees from the original electron beam and foil orientation.

\section{Resu1ts}

The creep and tensile data obtained from specimens from which our TEM microstructural observations were made, as well as the data of Howson et a1. on the same material heat (5), are plotted in Fig. 1b. All data points appear to be consistent with each other, and, as mentioned, the diffusion compensated strain rate versus modulus compensated stress plot shows a characteristic threshold stress below which no detectable creep occurs. The plot shows a very high stress exponent in the low stress regime which tends to lessen toward the higher modulus compensated stresses. In producing the plot, the diffusion and modulus values were taken from those discussed in the earlier paper on this subject (6).

The fine microstructural observations consistently showed the dislocations to be pinned at particles. Figure 2 includes representative TEM views from specimens crept and cooled on load at $760 \mathrm{deg}$. C and $1093 \mathrm{deg}$. C, respectfully. Note the dispersoid free zones are void of dislocations. Figure 3 shows typical sequences of views on specific dislocation-particle interactions at various stage tilts, revealing that dislocations maintain intimate contact with the particles though the dislocation appears to be on the departure side of the strengthening dispersoids. Such intimate 


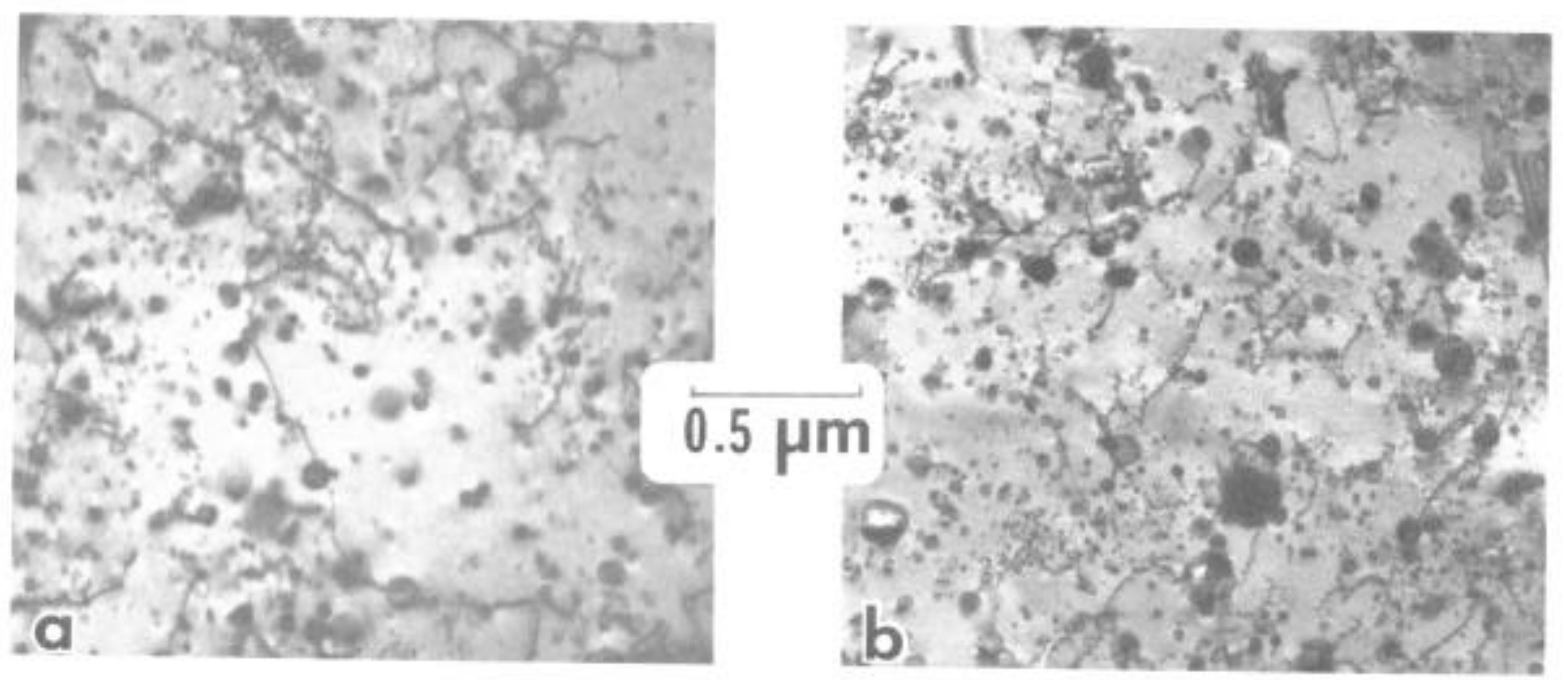

Figure 2 - Representative TEM micrograph of MA 754 deformed and cooled on load a) after tensile testing at $1093 \mathrm{deg}$. $C\left(\xi=1 \times 10^{-4} / \mathrm{s}\right.$ and $\left.\varepsilon=.02\right)$ and b) crept to steady state at $760 \mathrm{deg}$. C $\left(\dot{\varepsilon}=9 \times 10^{-8} / \mathrm{s}\right.$ and $\left.\varepsilon=.02\right)$, showing dislocations attractively pinned to the fine oxide particles.
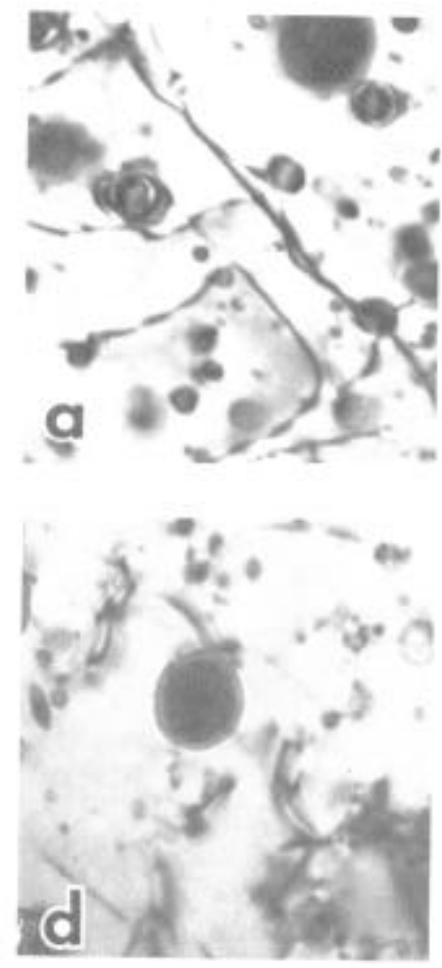

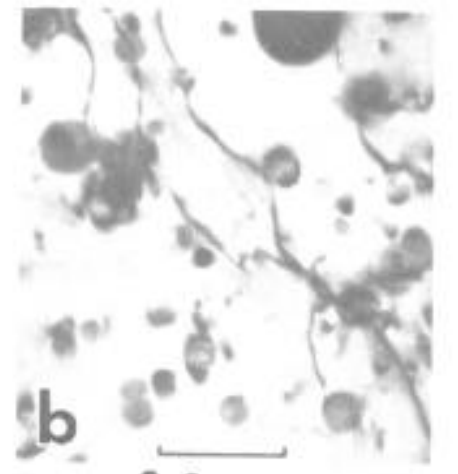

\section{$0.2 \mu \mathrm{m}$}

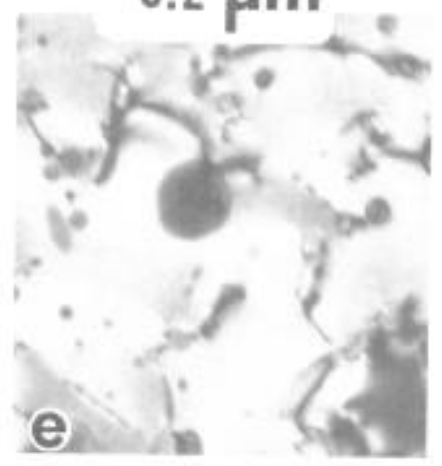

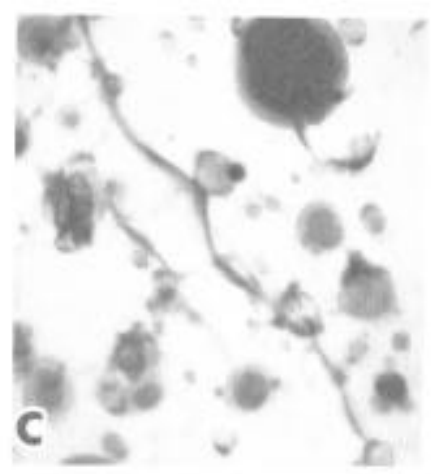

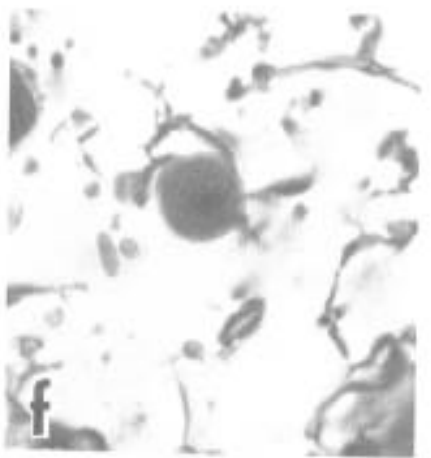

Figure 3 - Representative TEM micrographs of dislocations and specific particle interactions at a variety of specimen stage tilt 3 ; specimens were crept to steady state at $1093 \mathrm{deg}$. C $\left(\dot{\varepsilon}=1.28 \times 1 \mathrm{C}^{-5} / \mathrm{s}\right.$ and $E=0.01$ ) and cooled on load a) 0 deg. tilt, b) 20 deg., and c) 45 deg.; crept at $760 \mathrm{deg} . \mathrm{C}\left(\dot{\varepsilon}=2.15 \times 10^{-5} / \mathrm{s}\right.$ and $\left.\hat{E}=.02\right)$ and cooled on load d) 0 deg. tilt, e) 20 deg. and f) $50 \mathrm{deg}$. Note in both cases the dislocations are pinned on particles' departure sides, as evidenced by the fact that the dislocations are stuck on the sides oc the particles from which the islocations are bowing away. 
configurations were observed in thirty other foils from specimens tested. These observations are consistent with the existence of an attractive dislocation-particle interaction.

To further confirm the existence of an attractive interaction, dislocation configurations in specimens crept to steady state and then annealed off load were investigated. The dislocations still remained firmly attached to the particles, Figs. 4 and 5, even after loads were removed at the test temperature.

We observed an absence of any detectable Orowan loops around particles. The fact that no looping is seen does not in itself rule out repulsive interactions between dislocations and the oxide particles. Brown and Stobbs (3) have shown that the relaxation of loops around dispersoids could also account for their absence. The observed intimacy between dislocations and particles on the departure side of the particles, even after long time, offload annealing, is indisputable evidence of attractive interactions, however.

\section{Discussion}

Although the implications of attractive interactions between dislocations and oxide particles were not then recognized, that dislocations in ODS a1loys may be stuck to the oxide particles are discernible in electron micrographs appearing in past ODS related papers. TEM micrographs of TDnicke1 and TD-nichrome show such evidence in papers published as early as 1966. (10,11) The comprehensive paper published in 1980 (5) on Inconel MA 754 also contained micrographs showing strong residual pinning of dislocations to the oxide particles, much like those in the representative micrographs in this paper. The first proposed connection between creep thresholds and the observed strong attractive interaction of dislocations and oxide particles was made very recently, and independently, by Nardone and Tien (12) and by Srolovitz, et al. (13). The former paper (12) was accompanied by supporting TEM micrographs. Consequently, controversies did arise as to whether the dislocations were indeed stuck to the particles resulting in pinning even on the departure side of the particles. Arguments were made that the dislocations may instead be lying on planes above or below the particles and pinned by either the foil's free surfaces or sessile dislocations perpendicular to the electron view. According1y, the result from our careful and effort consuming tilting experiments are invaluable, since they represent indisputable evidence that there can be a strong attractive interaction between the mobile dislocations and the strengthening obstacles (the oxide particles). A dislocation line not really in contact with particles, may visually appear to be in contact in one view, but this illusion cannot be sustained through view tilting of over 45 degrees.

The reason for an attractive dislocation particle interaction must stem from a decrease in the energy of the system as a result of dislocation/particle association. The factors that can account for this decrease in energy are 1) a change in the line and core energy of the dislocation due to elastic modulus differences and 2) microchemistry effects at the oxide interface.

As mentioned, superficially, a decrease in the elastic energy of the dislocation seems unlikely since the polycrystalline elastic modulus of the oxide particles $\left(\mathrm{Y}_{2} \mathrm{O}_{3}\right)$ exceeds that of the fcc nickel-chromium matrix. Under these conditions, elasticity theory shows that a repulsive interaction should exist between the dislocations and the particles. The elastic 


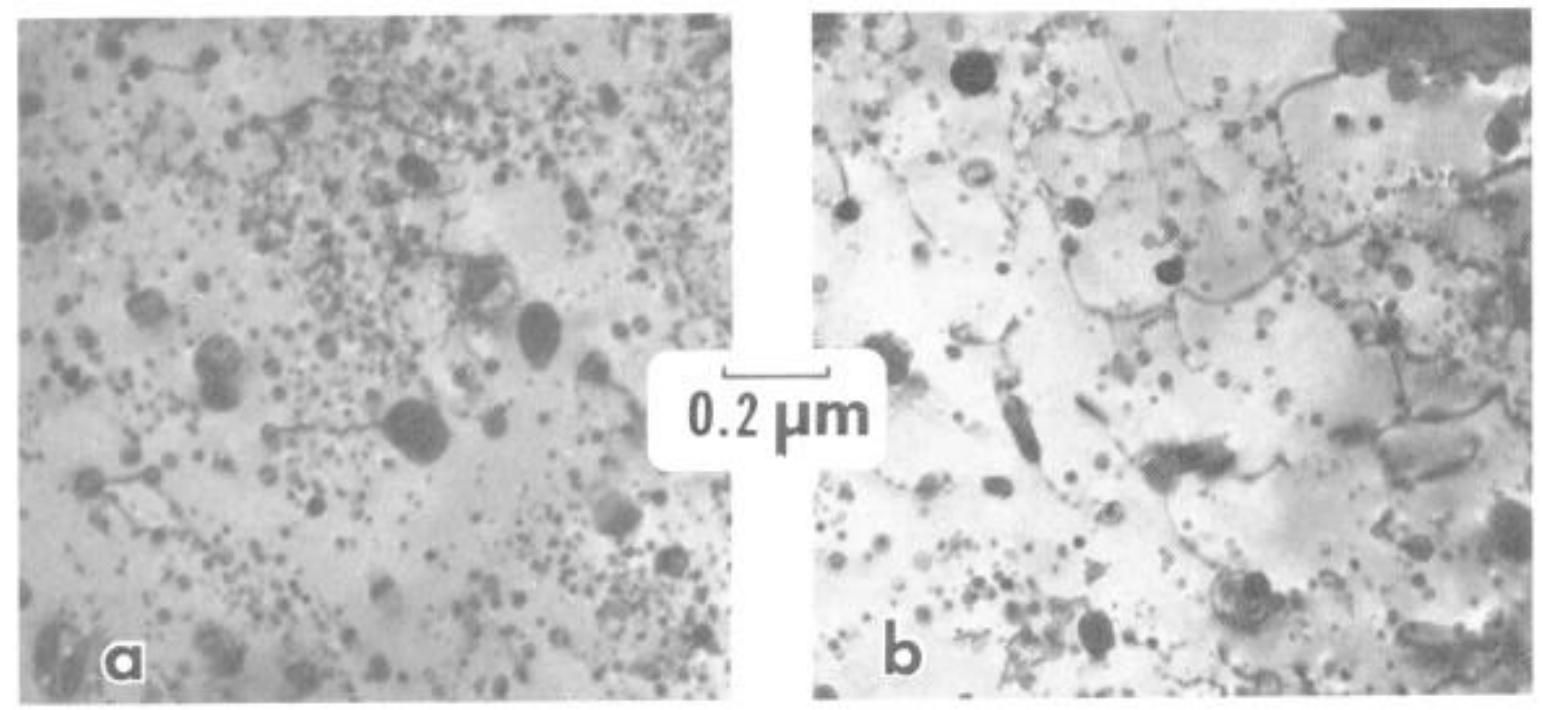

Figure 4 - TEM micrographs of MA 754 crept to steady state, unloaded and annealed, to allow dislocations to rearrange to lower energy configurations; a) $1093 \mathrm{deg} . \mathrm{C}$ and b) $760 \mathrm{deg}$. C. Note that the dislocations still maintain close contact with the dispersoids.
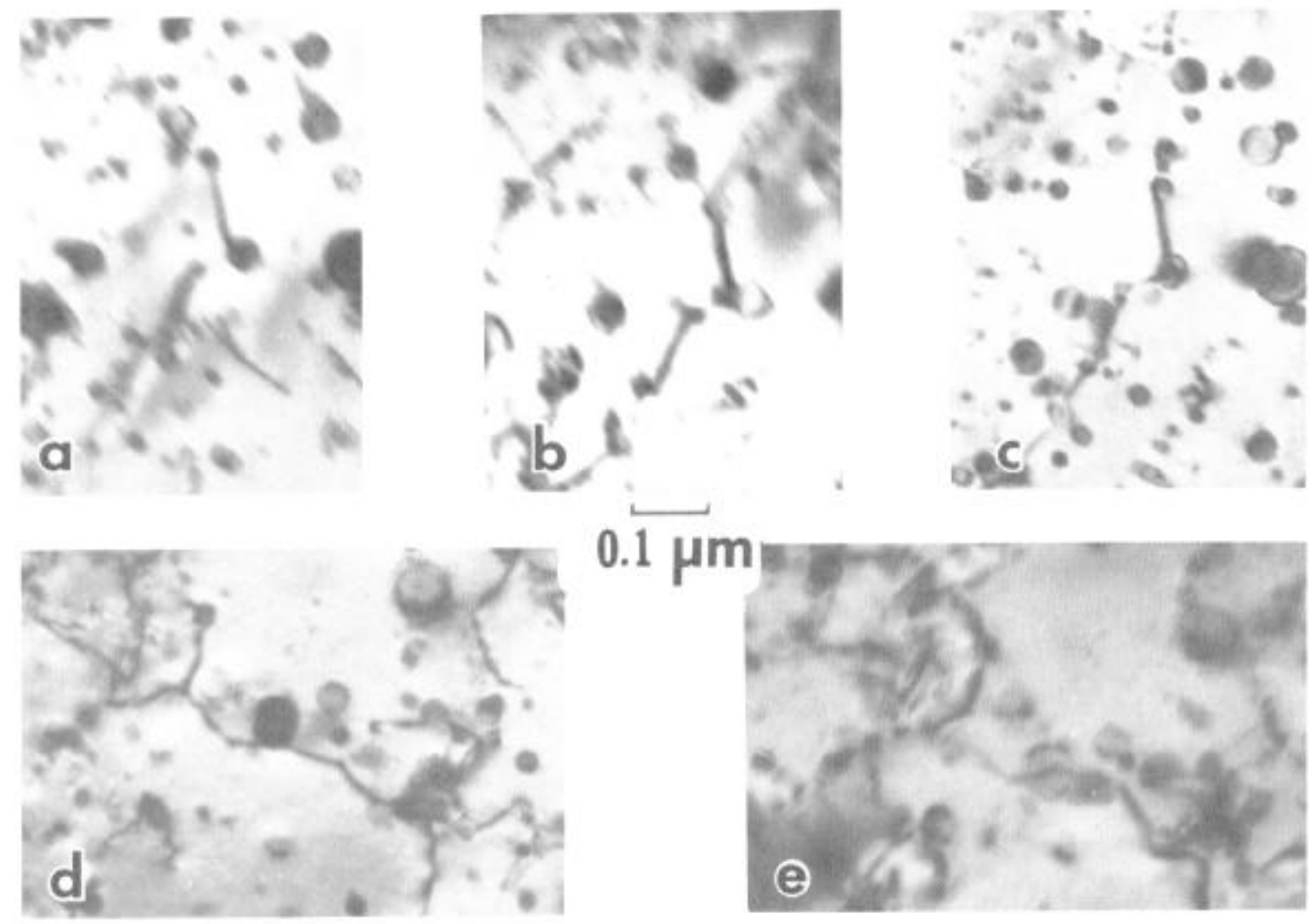

Figure 5 - TEM micrographs of MA 754 of dislocation-particle configurations at a variety of tilts; specimens were crept and then annealed off load at $1093 \mathrm{deg}$. C a) $0 \mathrm{deg}$. tilt, b) $20 \mathrm{deg}$. and c) $45 \mathrm{deg}$.; and at $760 \mathrm{deg}$. C d) $0 \mathrm{deg}$. tilt and e) $25 \mathrm{deg}$. . Note the dislocations still maintaining contact with the particles at all tilts shown. 
constants are very anisotropic in nature, however. For example, the 760 deg. C Young's modulus for textured MA 754 being $110 \times 10^{3} \mathrm{MPa}$, while the untextured material has a $760 \mathrm{deg}$. C Young's modulus of $154 \times 10^{3} \mathrm{MPa}$. (14) This confirms that there is a wide range of values for the elastic constants in a grain of Inconel MA 754. In addition, the oxides are essentially small single crystals, also having anisotropic elastic constants. At $760 \mathrm{deg}$. $\mathrm{C}_{3}$ the Young's modulus for polycrystalline $\mathrm{Y}_{2} \mathrm{O}_{3}$ was determined to be $145 \mathrm{x} 10^{3}$ $\mathrm{MPa}$. (15) Accordingly, there can be a wide range of relative orientations between oxide and matrix that can allow for an attractive dislocationparticle interaction, due to a decrease in the elastic energy of the dislocation.

The limit in attractive interaction is that between dislocations and voids, since such encounters result in the elimination of a segment of the dislocation line (and thus core and elastic energy) equal to the diameter of the void. It stands to reason then, regardless of the sign of the modulus difference between oxide particles and matrices, a maximum attractive interaction will occur if at temperature the oxide-matrix interface becomes nonadherent, separates and forms a localized minute void space - resulting effectively in a zero modulus particle. This idealized and hoped for extreme is not occurring in MA 754, however. As shown in the tilting sequence views in Figs. 3 and 5, dislocations that seem to have disappeared at particles were in fact pinned to the departure side of the oxide particles.

The exact nature of this pinning is not known at this time. (6) Certainly, trace impurities $(\mathrm{H}, 0, \mathrm{~N}, \mathrm{C}, \mathrm{S}$, etc.) in alloys will segregate and concentrate at the oxide/matrix interface. These or other impurities can then transfer on to the dislocation that is in intimate contact with the interface due to the afore-discussed and observed attraction between oxide particle and dislocation. Once stuck on the dislocations the impurities can pin the dislocation on the departure side of the dispersoids either through Cottrell-drag or by the lowering of the localized energy of the dislocation line segment that is wrapped about the particle. Dislocation escape then occurs by the applied stress bowing the dislocation which is now stuck at the departure sides of, say, two adjacent particles, until the line tension force exceeds the pinning forces due to the lower (localized) energy of the dislocation segment at the particles.

A detailed model for overcoming attractive dislocation-particle interaction has been derived (6), and the calculations show that a modest reduction of the stuck segment energy by only 5 percent is necessary in order to result in the threshold values of about 0.5 of the Orowan stress. Indeed, in the limit, for $100 \%$ reduction, i.e., in the wished-for non-adherent oxide/matrix interface case, it can be easily shown that the threshold value will then increase to be equal to the Orowan stress. Accordingly, attractive dislocation-oxide particle interaction precludes the limitation of a climb-modified lowering of the Orowan stress as the high temperature threshold strength. This means that the threshold in Fig. 1b, which corresponds to a stress of $94 \mathrm{MPa}$ at $1093 \mathrm{deg}$. C, can become as high as $188 \mathrm{MPa}$. Please note that a factor of two increase in threshold strength will result not on1y in a doub1ing of the fail-safe applied design stress, but will also result in a shift of the temperature normalized creep strain rate curve to the right (see dotted curve). As can be seen, Fig. 1b, such a shift will result in at least a two-orders-of-magnitude lowering of creep rates at applied stresses above the threshold. 


\section{Concluding Remarks}

The detailed TEM results of this study show beyond a reasonable doubt that the strengthening mechanism in such ODS, MA alloys as Inconel MA 754 is one of overcoming the attractive dislocation-oxide particle interaction. This type of strengthening is significantly different than the classical Orowan-type mechanism, which is based on repulsive dislocation-oxide particle interaction. Accordingly, the high temperature strength and creep resistance of the ODS alloys can be altered and improved by altering not only interparticle spacing (particle size and volume fraction), but also by manipulating the adherence of the dispersoid/matrix interface, and by local chemistries. Indeed, we show that the results strongly suggest that the infinite-1ife threshold creep strength can be significantly raised and the creep rates at higher stresses lowered by orders-of-magnitude if the interface is made non-adherent resulting in effect in a circumferencial void space.

How this can be accomplished requires, of course, further research into adsorbates, expansion coefficients, interfacial strength, and especially the matrix and oxide bonding steps during mechanical alloying. The results of this paper just underscores the need, and define the potential gains if it can be accomplished.

\section{Acknowledgements}

We thank the Materials Science Division of the NSF for supporting this work under Grant DMR 80-11402. Dr. Robert Reynik was the Program Manager of this Grant. Inconel MA is an alloy designation of the International Nickel Family of Companies. We wish also to thank INCO for fellowship support of Ms. Cooper.

\section{$\underline{\text { References }}$}

1. E. Orowan, Discussion in The Symposium on Internal Stresses in Metals and Alloys, 1948, p. 451.

2. R. S. W. Shewfe1t and L. M. Brown, "High-temperature Strength of Dispersion-Hardened Single Crystals," Philosophical Magazine, 35 (4) (1977) pp. 945-962.

3. L. M. Brown and W. M. Stobbs, "Modelling Structural Changes in Deformed Dispersion Strengthened Crystals," pp. 387-431 in Constitutive Equations in Plasticity, Ali S. Argon, ed.; MIT Press, Cambridge, MA, 1975.

4. R. W. Lund and W. D. Nix, "High Temperature Creep of Ni-2OCr-2ThO Single Crystals," Acta Metallurgica, 24 (1976) pp. 469-481.

5. T. E. Howson, J. E. Stulga and J. K. Tien, "The Creep and Stress Rupture of Oxide Dispersion Strengthened Mechanically Alloyed Inconel MA 754," Met. Trans. A, 11A (1980) pp. 1599-1607.

6. V. C. Nardone, D. E. Matejczyk and J. K. Tien, "The Threshold Stress and Departure Side Pinning of Dislocations by Dispersoids," accepted for publication in Acta Met., 1984.

7. G. M. Pharr and W. D. Nix, "A Comparison of the Orowan Stress With the Threshold Stress for Creep for Ni-20Cr-2ThO 2 Single Crystals," Scripta Met. 10 (11) 1976, pp. 1007-1010. 
8. R. Petkovic-Luton, D. J. Srolovitz and M. J. Luton, "Microstructura1 Aspects of Creep in Oxide Dispersion Strengthened Alloys," presented at INCOMAP Conference on MA Alloys, London, July 1983, INCO publisher, Sterling Forest, N.Y.

9. R. R. Jensen and J. K. Tien, "Creep and High Temperature Deformation of Simple Metals and Superalloys," pp. 529-551 in Metallurgical Treatises, J. K. Tien and J. F. E1liott eds.; AIME, Warrendale, PA, 1981.

10. B. A. Wilcox and A. H. Clauer, "Creep of Thoriated Nickel Above and Below $0.5 \mathrm{~T}_{\mathrm{m}}$," Transactions of the AIME, 236 (1966) pp.570-580.

11. J. H. Hausse1t and W. D. Nix, "Dislocation Structure of $\mathrm{Ni}-2 \mathrm{OCr}-2 \mathrm{ThO}_{2}$ After High Temperature Deformation," Acta Met., 25 (1977) pp. 595-607.

12. V. C. Nardone and J. K. Tien, "Pinning of Dislocations on the Departure Side of the Strengthening Dispersoids," Scripta Met., 17 (4) (1983) pp. 467-470.

13. D. Srolovitz, R. Petkovic-Luton and M. J. Luton, "On DislocationIncoherent Particle Interaction at High Temperatures," Scripta Met., 16 (1982) pp. 1401-1406.

14. INCOMAP Mechanica11y A1loyed Products Engineering Data, Inco Limited, One New York Plaza, New York, N.Y., 1981.

15. W. R. Manning and O. Hunter, Jr., "Elastic Properties of Polycrystalline Yttrium Oxide, Holmium Oxide, and Erbium Oxide: High-Temperature Measurements," Journal of the American Chemical Society, 52 (9) (1969) pp. 492-496. 